\title{
MEASURING THE PERFORMANCE OF HANDOFF IN HETEROGENEOUS WIRELESS NETWORKS USING NETWORK LAYER PROTOCOL
}

\author{
Sasikala T. ${ }^{1}$ and Srivatsa S.K. ${ }^{2}$ \\ ${ }^{1}$ Research Scholor, Sathyabama University, Jeppiaar Nagar, Rajiv Gandhi Road, Chennai, India \\ ${ }^{2}$ St.Joseph's College of Engineering, Rajiv Gandhi Road, Chennai, India \\ E-mail : 'sasi_madhu2k2@yahoo.co.in
}

\begin{abstract}
Future wireless Internet access will be dominated by $3 \mathrm{G}$ wide area cellular networks and hot spot wireless LANs. Wired connections usually provide high speed, reliable access to the Internet; wireless network technology enables users to access customized Internet services even when they are moving. If a user wishes to have an uninterrupted Internet service, while frequently switching from one network connection to another, a seamless handoff solution with low latency and low packet loss is mandatory [Ling-Jyh Chen et al]. Wireless technology eradicated the limitation of users mobility and device portability. Among various wireless access technology wireless LAN and cellular network have turned out to be the most widely deployed infrastructures providing mobile access to voice and data services upon users needs. Moving from one subnet to another causes a handoff. During handoff, a mobile node point of attachment to the fixed network is transferred to another. This causes a disruption in the ongoing data flow. If handoff is not handled properly, it causes user perceivable degradation of quality of service. We have analyzed and compare different protocol for handoff, by introducing new features.
\end{abstract}

Key words: WLAN,UMTS, Handoff, MIP, mSCTP

\section{INTRODUCTION}

The main challenges during integration of the two wireless services are, inter working architectures, billing issues, mobility and roaming, security/authentication and session persistence. There are two different ways of integrating two wireless technologies, called loosely coupling and tightly coupling. The billing of the customer is an important factor in the integration of two wireless networks. The functionality of the billing mediator is to convert accounting statistics from both the networks into a format native to the particular billing system used by the operator. With seamless roaming, the end user will not be aware of any change in the network they are using and will not be required to interact with the system to enable handoff [Ronan Morrissey et al .2002]. The major problems of wireless communication are higher error rates, lower bandwidth and more frequent spurious disconnection. As a result of these factors, communication latency rises due to higher retransmission, retransmission time-out delay increases, and more error-control protocol processing is required.

\section{RELATED WORK}

Lima and Tejinder describes Vertical handoff using SCTP by having Client Server model (i.e. between the machine and the fixed server). They use only loosely coupled architecture. The performance metrics they considered is only handoff delay and through put. (Mohammed Jaseemuddin) has proposed architecture of integrating UMTS and 802.11 WLAN, which allows a mobile node to maintain data (packet switched) connection through WLAN and voice (circuit switched) connection through UMTS in parallel. Seamless handoff of mobile terminal from WLAN to CDMA 2000 network was described by (Hemish Parikh et al) using Proactive and spurious handoff triggers. (Shiao - LI Tsao et al) describes three possible UMTS -WLAN inter networking strategies. They got very poor performance $(200 \mathrm{~ms}$ latency and packet loss) while the user is more than 2000. (Hecto velayos et al) has given the technique to reduce the IEEE802.11b handoff time using 3 phases. They found that the search phase could be reduced by $20 \%$ compared to the shortest measured one.

(Leo patina pongpibul et al, 2003) have developed a test bed for client based handoff mechanism for mobile IPV6 wireless network. They said that their mechanism reduces the handoff latency of TCP connection by $50 \%$. (Peter De Cleyn et al) presents a smooth handoff scheme using 802.11 IEEE triggers together with packet buffer in the old AP and packet forwarding from the old to the new AP. They described a technique to reduce the link layer handoff time. They found that handoffs are completely managed at layer 3.A soft handoff procedure between 2 wireless LAN using Ethernet switch is explained by [Raheem A Beyah et al) to ensure optimal performance, minimal service description, as well as minimal broadcast frame. They also said to implement the same concept in cellular network. (Sharjian et al) had worked on TRASH, a new transport layer seamless handoff for mobile network and they considered various aspects such as handoff signaling, location management, data transfer and security considerations using multi homing feature of 
SCTP .The focus of this work is to measure the performance of the handoff using handoff rate as a parameter.

\section{BENEFITS OF THIS RESEARCH}

Integration of wireless networks can bring significant benefits to service providers and end users. Also, the customers will benefit from enhanced performance through greater coverage, higher data rate, increased efficiency, higher productivity and lower overall cost of such a combined service. It also allows a system wide data to exist in a common database, which simplifies data reporting and storage [www.dfm-net.dk]. WLAN make use of unlicensed free frequency band to provide low cost, high data rate wireless services in hotspots area such as airport, restaurant etc. Thus, the integration of two systems can combine their best features to provide ubiquitous access while overcoming weaknesses of both systems. One major challenge in seamless integrating WLAN with UMTS cellular networks is the design of reliable, robust, and efficient internetworking architecture [Chunming Liv et al 2005.]. $3 \mathrm{G}$ networks are expected to include capabilities and features such as, enhanced multimedia, broad bandwidth, routing flexibility and true global roaming capability.

\section{METHODOLOGY ADOPTED}

As shown in the figure 1 WLAN in the hotspot is a closed room, where one or two entrance is available for entry and exit. So a mobile user could move out and move in only via that entrance. We have set the flags in the entrance as 1 ,and the other sides of the room as 0 . There are two possibilities of occurring handoff here. They are:

i) The mobile user is moving towards the entrance of the hotspot from the WLAN. Instantly the signal strength of the mobile node is getting decreased. So, an inter technology handoff is initiated by the mobile node between WLAN and UMTS networks.

ii) A mobile user from UMTS is moving towards the hotspot entrance. Even if the signal strength is strong enough, in order to utilize the bandwidth of the network, it is necessary to have a handoff from UMTS to WLAN.

To solve this problem a handoff decision criterion is designed for the mobile users.

\section{Criterion for WLAN to UMTS}

\section{Handoff}

1. Mobile node is in WLAN

2. Assign integer values to $X$
3. Check the flag.

4. IF flag $=1$ then

5. Measure the RSS

6. If RSS $>=X$ then wait for $T 1$ second $G$ o to step 5 . Else initiate Vertical handoff from WLAN to UMTS networks.

7. Else// less chances of initiating handoff

8. Continue in WLAN itself

\section{Criterion for UMTS to WLAN handoff}

1. Mobile user is in UMTS network.

2. Measure the RSS

3. Periodically check whether the MN is near to WLAN network or not.

4. Check the flag

5. If the flag=1 and RSS $>=X$ initiate inter technology handoff from UMTS to WLAN // In order to Utilize the maximum bandwidth.

6. Else continue in the UMTS network itself.

We are using a dual mobile mode terminal with both WLAN and UMTS interface. The software inside the dual mobile mode can switch between WLAN and UMTS networks, depending upon the availability of networks. Here the WLAN network is within the coverage area of the UMTS networks.

When the user is in UMTS networks, and it is near to the coverage area of the WLAN (i.e. when it receives the beacon signal), the WLAN MAC layer of the dual mobile node computes the signal strength of the received signal. Even if the signal strength is greater than the threshold value, the mobile node initiates the handoff, in order to utilize the maximum bandwidth. It is called as Soft handoff.

When the mobile node moves out of the coverage area of the WLAN network, the WLAN interface detects the reduced strength of the signal and gives the information to the dual mobile node software. Immediately the mobile node initiates the handoff from WLAN to UMTS networks. It is also called as hard handoff .Now the information is accessed from the Internet via UMTS networks [Siddiqui et al 2005]. 


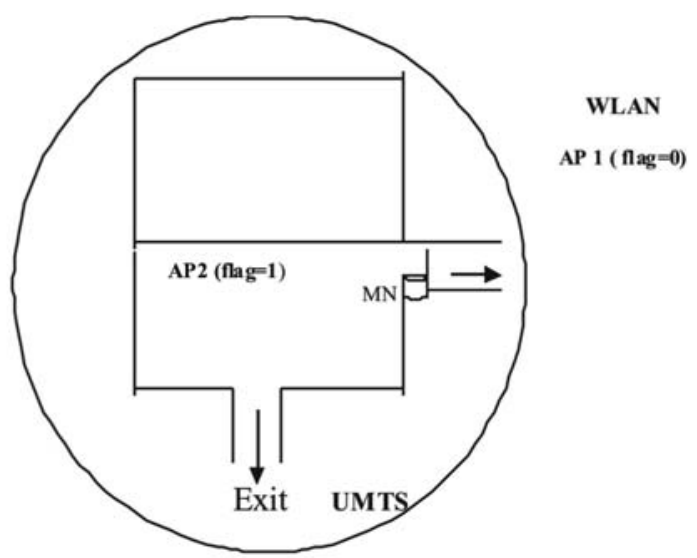

Fig .1 Scenario used for the integrated environment

We have used two protocols to measure the performance of handoff. They are MIP and MSCTP. Handoff using MIP protocol is described here.

\section{MIP Protocol}

Here the method adopted is terminal initiated handoff [Cheng et al 2005.]. WLAN and UMTS are entirely different technologies. Since this work uses infrastructure based WLAN, it is directly connected to Internet via access point. Based on the network layer mobility, MIP (Mobile IP) became a standard protocol for the mobile users. In view of its registration and authentication procedures, this protocol suffers significant handoff delay and packet loss. Our work introduces some enhancement procedures to increase the performance of MIP. When the rate of $\mathrm{MN}$ (Mobile Node) movement increases, the registration of $\mathrm{MN}$ with a home agent increases. This will causes a high handoff delay. In order to reduce this, new agent was defined [lan et al 2004, Eva et al 2004, Hesham Soliman et al 2004.].

The steps involved will be agent discovery, authentication, registration and handoff,which is described in the Fig .2

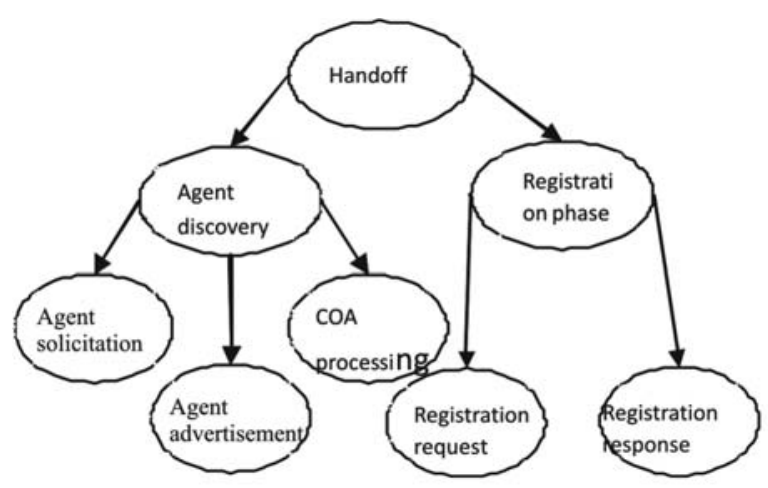

Fig .2 Data flow diagram for handoff delay
We have used the TCP segments for data transfer. The delay, packet loss, end to end throughput are calculated using the following formulas.

Handoff delay (MIP)=Agent Discovery

Period

$+$

Registration

period.

End to end Throughput (MIP) $=$

(Total Traffic offered-Total data loss)/Total transmission duration

Packet loss (MIP) =

Sum of data loss incurred by each handoff/Packet size

\section{SIMULATION RESULTS AND DISCUSSIONS}

To test the performance of the integrated network architecture, the work is implemented in NS2 2.26 version. The simulated results are shown in Figure 3 through 5.The handoff rate is the number of handoffs during the simulation in unit time.

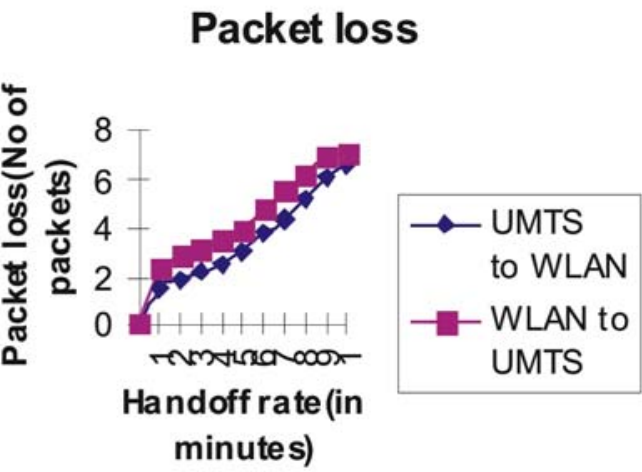

Fig .3 Packet losses in UMTS-WLAN 802.11b network and vice versa

The Fig .3 shows the number of packets loss over the handoff rate. As because of the higher bandwidth of WLAN, packet losses are more, when the user moves from WLAN to UMTS networks. 


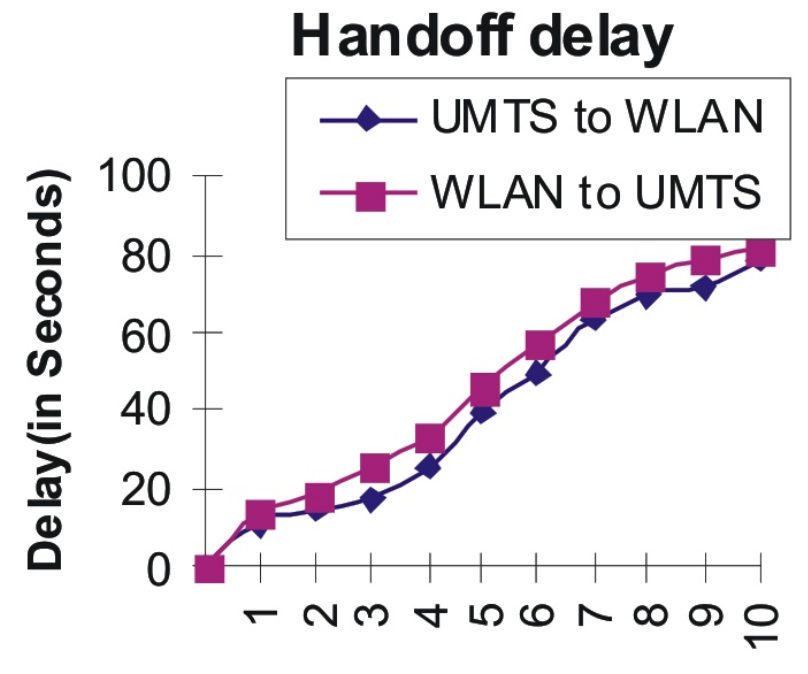

Handoff rate(in minutes)

Fig .4 Handoff delay in UMTS-WLAN802.11b network and vice versa

Fig .4 shows that, due to the registration procedure of MIP, there is an increase in delay both from the network. As WLAN to UMTS is using the hard handoff, there is more delay during the transition than from UMTS to WLAN network. Fig .5 shows clearly that end-to-end throughput of MIP decreases in WLAN to UMTS network than vice versa.

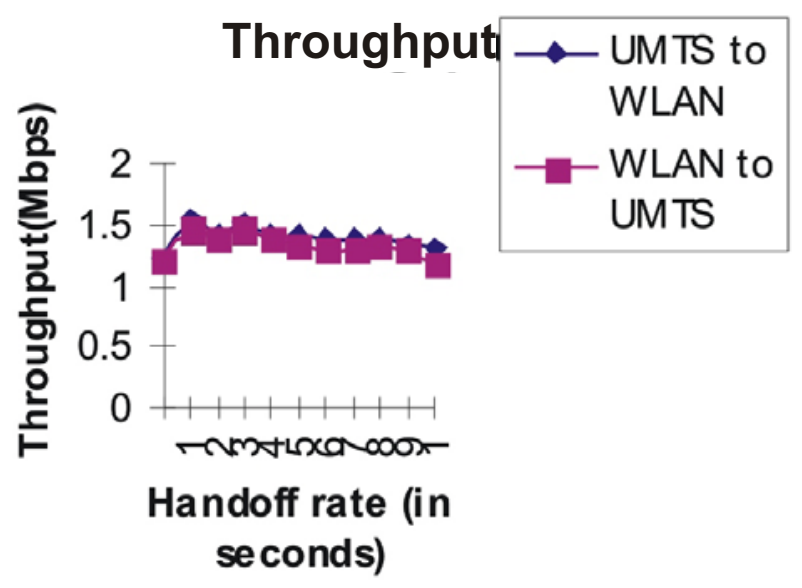

Fig .5 End to end throughput UMTS-WLAN $802.11 \mathrm{~b}$ network and vice versa

\section{CONCLUSION}

Both WLAN and UMTS can be viewed as competing and or complementary technologies. There fore an integration of WLAN $s$ with UMTS will bring advantages to the users as well as the service providers. We have considered the problem of seamless handoff of WLAN and UMTS networks. A novel scheme is proposed to improve the handoff performance. The proposed handoff scheme uses a MIP and MSCTP protocols for implementation. The analysis of performance of the MIP scheme was only presented in this paper. Although currently this scheme is verified only with IEEE802.11b and UMTS networks, it does not depend on any specific protocols details. Our proposed scheme reduces the signaling overhead on the Internet and minimizes packet loss for the mobile node during handoff.

\section{REFERENCES}

[1] Ronan Morrissey, Julian Leonard and Neil O'Driscoll(2002),"UMTS (3G)/WLAN Integration",Department of Electronic and Computer Engineering,University of Limerick ,Ireland

[2] Shiao-Li Tsao and Chia-Ching Lin(2002),"Design and Evaluation of UMTSWLAN Internetworking Strategies",pp 777-781.

[3] Cheng Wei Lee,Li Ming Chen, Meng Chang Chenand Yeali Sunny Sun(2005),"A frame work of handoffs in Wireless Overlay Networks Based on Mobile Ipv6", Areas in communications,Vol.23,No.11.

[4] Li Ma,Fei Yu,victor C.M.Leung,and Tejinder Randhawa(2004),"A new method to support UMTS/WLAN vertical handover using SCTP",IEEE wireless communications,pp44-51.

[5] Siddiqui,S.Zeadally,S.Fowler(2005),"A novel architecture for roaming between $3 G$ and wireless LANs",IEEE,0-7803-9319-8/05.

[6] Hesham Soliman,Claude Catelluccia, Karim E1 Malki and Ludovic Beller,"Hierarchical mobile I pv6 mobility management" (hmipv6): http://www.ietf.org/ proceedings/04aug/l-d/draft-ietf-mipshophmipv6-02.txt.

[7] Eva Gustafsson,Annika Jonsson,and Charles E.Perkins(2004),"Mobile Ipv4 regional registration":http://www.ietf.org/proceedings/04 aug/l-d/draft-ietf-mobilelP-reg-tunnel-09.txt.

[8] Muhammad Jaseemuddin(2003),"An Architecture for integrating UMTS and 802.11 WLAN Networks", Dept. of Electrical and Computer Engineering, Ryerson University,pp 716-723. 
[9] Shaojian Fu,Mohammed Atiquzzaman,Liran Ma,William Ivancic,Yong-Jin Lee,Justin S.Jones and Song Lu(2004)," TraSH:A Transport Layer Seamless Handover for Mobile Networks",University of Oklahoma,Norman,OK 73019-6151,USA,OU-TNRL-04-100.

[10] Ian F. Akyildiz, Jiang Xie, and Shantidev Mohanty(2004),"A survey of mobility management in next-generation all-ip-based wireless systems", IEEE wireless communications,pp 16-28.

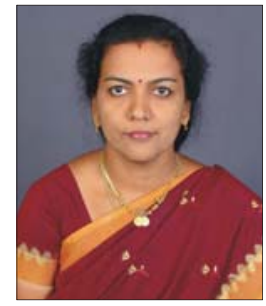

Ms. Sasikala - Assistant Pofessor, Research scholar, Department of Computer Science and Engineering, Sathyabama University. She has presented 6 papers in national and international conferences and published 3 papers in journals. She has 9 years of academic experience and her area of research is Networking. 